Щ्)FRANÇAISE

$\gg \mathrm{DE}$

필 PEGOGIE

\section{Revue française de pédagogie}

Recherches en éducation

190 | janvier-février-mars 2015

La formation des adultes, lieu de recompositions?

\title{
Les transformations des relations entre travail, éducation et formation dans l'organisation sociale contemporaine : questions posées par trois dispositifs analyseurs
}

The transformations of the relations between work, education and training in the contemporary social organization: questions asked by three analyzing devices

\section{Patricia Champy-Remoussenard}

\section{CpenEdition}

Journals

Édition électronique

URL : http://journals.openedition.org/rfp/4675

DOI : $10.4000 / \mathrm{rfp} .4675$

ISSN : 2105-2913

Éditeur

ENS Éditions

Édition imprimée

Date de publication : 31 mars 2015

Pagination : 15-28

ISBN : 978-2-84788-768-6

ISSN : 0556-7807

Référence électronique

Patricia Champy-Remoussenard, « Les transformations des relations entre travail, éducation et formation dans l'organisation sociale contemporaine : questions posées par trois dispositifs analyseurs », Revue française de pédagogie [En ligne], 190 | janvier-février-mars 2015, mis en ligne le 31 mars 2018, consulté le 03 janvier 2020. URL : http://journals.openedition.org/rfp/4675; DOI : $10.4000 /$ rfp. 4675 


\title{
Les transformations des relations entre travail, éducation et formation dans l'organisation sociale contemporaine : questions posées par trois dispositifs analyseurs
}

\author{
Patricia Champy-Remoussenard
}

\begin{abstract}
Cet article propose de saisir ce qui caractérise actuellement les relations entre l'éducation, la formation et le travail en s'intéressant aux évolutions du champ des activités professionnelles. Les termes en usage pour désigner ces faits sociaux, les évolutions les plus sensibles dans le champ professionnel, la quête d'adéquation entre formation et emploi et les logiques de professionnalisation, de compétences et d'évaluation peuvent être analysés à partir du développement de trois types de pratiques associés à des dispositifs : les stages en cours de formation, la validation des acquis de l'expérience, le mode entrepreneurial de l'activité et l'éducation à l'esprit d'entreprendre. Ces pratiques et dispositifs sont propres à analyser, sous différents angles, les évolutions, souvent en tensions, de ce que nous envisagerons comme des aspects de la vie sociale interdépendants : le champ des activités professionnelles, la forme scolaire, les compétences, et les logiques de professionnalisation.
\end{abstract}

Mots-clés (TESE) : environnement économique (économie, travail et emploi), politique et administration de l'enseignement, contenu de l'éducation, environnement social (société, culture, langues et religion).

\section{Introduction}

Qu'est-ce qui caractérise actuellement les relations entre éducation, formation et travail ? Dans une société où chacun est appelé à apprendre tout au long de sa vie (lifelong learning), le champ éducatif comprend toutes les pratiques qui visent l'acquisition des différents savoirs, l'accompagnement du développement des individus et des collectifs. Nous proposons ici un certain nombre de pistes d'analyse des évolutions les plus sensibles dans le champ des activités professionnelles en interrogeant trois dispositifs propres à saisir 
les évolutions des faits sociaux interdépendants que sont les pratiques d'éducation et de formation et celles du travail (Barbier, 2001).

Pour commencer, une caractérisation d'un point de vue sociologique et critique des principales tendances d'évolution du travail montrera leurs influences potentielles sur l'éducation et la formation. Puis nous ferons le point sur la recherche d'adéquation entre formation et emploi, les logiques de professionnalisation, de compétences et d'évaluation. Enfin nous interrogerons plus précisément trois dispositifs en cours de développement (l'accès à la certification par la validation des acquis de l'expérience, le développement des stages en cours d'études, l'éducation à l'esprit d'entreprendre et le mode entrepreneurial de l'activité) en les saisissant comme des analyseurs des actuelles tendances de reconfiguration des relations entre travail/ éducation et formation. Relativement récents, ils permettent de comprendre, à partir de pratiques émergentes, les transformations sociales les plus marquées aujourd'hui, et de repérer leurs paradoxes et les tensions qu'elles font vivre aux acteurs ${ }^{1}$.

\section{Un état des transformations des relations entre travail et formation au début du $x{ }^{\mathrm{e}}$ siècle}

Une des caractéristiques de la production de connaissance sur les activités professionnelles et sur les activités d'éducation et de formation est qu'elle s'opère dans une confusion entre discours ordinaires, politiques, institutionnels, et termes et concepts scientifiques. Naville (1954) signalait cette difficulté épistémologique associée au double usage de mots tels que «travail». Ceux-ci sont en effet toujours à la fois présents dans les discours de l'opinion (Bachelard, [1934] 2000) et les énoncés de croyance (Marie, 2011) et mobilisés dans l'outillage conceptuel et méthodologique de la production de connaissances sur le travail. Martuccelli $(2010$, p. 8) remarque lui aussi la tendance des analystes à utiliser des termes «jamais en rupture radicale avec l'expérience sociale.»C'est pour dépasser ces difficultés que Barbier (2011, p.11) propose de distinguer lexique de l'action et lexique de l'intelligibilité de l'ac-

1 Les questions abordées nécessitent de faire des choix conceptuels entre différents auteurs ou courants qui ne pourront pas être développés ici. tion et, à la suite, concepts mobilisateurs et concepts d'analyse ou d'intelligibilité.

L'espace social est ainsi saturé par l'usage incessant des termes «compétence», «emploi», «professionnalisation» ou encore «expérience», «qualification», "performance», «employabilité», «flexibilité», «flexicurité», «mobilité », "sécurisation», "précarité», etc. qui nous informent sur des évolutions souhaitées ou effectives du rapport à l'activité professionnelle et qui circulent tout à la fois dans le champ des pratiques de formation et dans celui des recherches sur l'éducation, la formation et le travail.

Ainsi, dénommées, un ensemble d'évolutions caractérisent tendanciellement la société contemporaine, se répercutent sur les activités humaines, notamment au travail et en formation, et redessinent les relations entre éducation, formation et travail. À titre exploratoire, nous nous attarderons sur certaines d'entre elles : le statut de l'activité de travail (sa centralité, son utilité et sa rentabilité), son potentiel infini de développement, le modèle de la flexibilité et les redéfinitions des rapports des individus au temps, aux distances et aux collectifs.

\section{Centralité du travail}

Les analyses relatives à la centralité du travail déjà présentes chez Marx, puis poursuivies dans les années 1960, 1970 (Naville, 1963 ; Balibar, 1968, et plus récemment Méda, 1998; Barel, 1989 ; Zarifian, 1995, etc.), défendent I'hypothèse selon laquelle, dans I'organisation globale des activités humaines, l'activité professionnelle est un «pivot de la distribution des revenus, des droits et des protections» (Méda, in Grillot, 2013). Même quand des individus sont privés d'activité professionnelle, leur position/situation sociale se définit à partir du rapport (de distance ou de proximité) au travail. Les injonctions et les préoccupations sociales de professionnalisation (Wittorski, 2008) des pratiques d'éducation et de formation s'accordent en quelque sorte à ce principe structurant, et en expliquent le succès social. Dans notre société, celui qui n'a pas d'emploi est contraint de rechercher de l'activité et de faire la preuve qu'il y consacre du temps et de l'énergie. La notion d'employabilité traduit cet ajustement exigé de l'individu. Les catégories administratives reflètent le caractère central attribué au travail. Les termes et notions de population active et de vie active témoignent des tensions dans lesquels sont pris les acteurs. La population active regroupe la «population active ayant un emploi » et les 
chômeurs ${ }^{2}$, mais aussi les personnes qui déclarent «aider une personne dans son travail (même sans rémunération)» alors que "ne sont pas retenues les personnes qui, bien que s'étant déclarées chômeurs, précisent qu'elles ne recherchent pas d'emploi ». N'est donc pas considéré comme actif celle ou celui qui ne fait pas la preuve de l'engagement dans un projet d'activité professionnelle.

Une part de cette centralité tient aussi au fait que les activités de travail en tant qu'expériences sont traversées par des apprentissages et génèrent des acquis. Elle n'est donc pas exclusivement conjoncturelle et propre à un contexte socio-historique mais aussi dépendante du fait que le travail est «formateur».

\section{Utilité et rentabilité du travail}

Dans les pays dits "développés», l'activité est également définie par son inutilité ou son utilité et son potentiel de création de valeur. La valeur ajoutée - indicateur économique - mesure la richesse créée au cours d'une période donnée, et l'activité, pour être considérée comme rentable, doit être productrice de valeur, donc de profit. Profit et rentabilité financiers constituent des valeurs centrales qui gouvernent en partie I'organisation de la vie sociale et font irruption dans des univers d'où elles étaient préalablement absentes. Des institutions du champ de la santé, du travail social, de l'éducation sont ainsi appelées à se conformer à des objectifs de rentabilité allant dans le sens d'une économicisation de leur fonctionnement (Bongrand, 2012). L'utilité sociale est un autre facteur de légitimité de l'activité qui doit générer un service rendu à la société et être au service de ses besoins. Tantôt qualifiée de publique, de sociale, d'économique, elle relève d'une construction sociale et évolue en permanence. La double jauge de l'utilité et de la rentabilité règle pour partie la hiérarchisation des activités en termes de reconnaissance (salariale, médiatique, etc.). Une incompatibilité temporelle entre deux registres d'action explique les insolubles paradoxes qui font le travail actuel : un registre utilitariste basé sur la recherche rationnelle et explicite de bénéfices égoïstes et des règles de donnant-donnant, un registre anti-utilitariste relevant de l'altruisme (Imbert \& Durand, 2014). La recherche d'utilité et de rentabilité de l'activité se

2 Le Bureau international du Travail, les services de la comptabilité nationale et les services du recensement français s'accordent sur ce paradoxal amalgame (2009). répercute en effet sur les conceptions et les pratiques de formation. Elle constitue une des déclinaisons de I'intention sociale de professionnalisation et un de ses fondements. C'est ainsi que se développent de plus en plus les formations de courte durée en lien étroit avec une utilité directe dans le milieu de travail qui cantonnent la formation à la flexibilisation du marché du travail, sans réelle sécurisation des trajectoires ni accroissement des capacités des individus (Conter \& Orianne, 2011). Enfin, c'est la valeur conférée aux titres et aux diplômes, et donc le processus d'attribution des certifications, qui évoluent eux aussi en lien avec les fluctuations de valeurs attribuées à certaines activités et leur inégale rentabilité (Maillard, 2013).

\section{Le potentiel infini d'action}

Pour Martuccelli (2010, p. 101), I'acteur de la société contemporaine est considéré comme « toujours capable d'agir». Les technologies numériques rendent en effet possible très rapidement le traitement de données multiples et complexes et la simultanéité des activités. L'individu est alors non plus défini par sa force de travail mais par son potentiel d'activité, sa capacité générique d'action (sa rapidité et sa puissance) (Martuccelli, 2010, p. 103). Les termes d'hyperactivité, de surcharge de travail, d'activisme, etc. reflètent cette conception de l'activité en tant que potentiel quasi illimité. L'individu et les collectifs finissent cependant par se trouver dépassés. La gestion des échanges par messageries électroniques est un exemple qui montre comment la puissance des modes de communication et d'échanges d'information reconfigure de manière non maîtrisable et quasi inhumaine les activités humaines (Monceau, 2013). Même le jeune enfant peut être hyperactif, comme en témoigne la palette des multiples activités qui lui sont proposées et le débat sur les rythmes scolaires ${ }^{3}$. Le temps «libéré» par le travail tout comme par le travail scolaire doit être occupé par des activités de loisirs.

Ce nouveau rapport à l'activité génère tout à la fois plaisir et souffrance. Face à l'épreuve, l'acteur souffre, résiste, s'efforce de survivre (Delory-Momberger \& Niewiadomski, 2009). La recherche de l'excellence et de l'exploit, les success stories et la performance côtoient les burn out et les dépressions. Dépassé par son potentiel d'action, l'individu s'épuise et se marginalise. Les

3 Qui masque, selon nous, le débat sur l'équilibre entre les activités des différents acteurs dans la vie sociale et sa relation avec l'organisation du temps scolaire. 
risques psychosociaux désormais repérés, dénommés, génèrent une activité d'accompagnement et de formation, avatars d'une société où l'activité confronte au risque de multiples manières (Beck, 1986).

\section{La flexibilité et le risque comme modèles de rapport à l'activité}

Les termes employabilité, flexibilité, mobilité, autonomie, initiative... omniprésents traduisent «toutes les attentes focalisées sur le salarié » quand adaptation, et adaptabilité résumeraient «le comportement, la posture morale qu'on attend de lui» (Linhart, 2009) en légitimant parfaitement les logiques à l'œuvre dans les activités d'éducation et de formation. La logique du lifelong learning, en véhiculant l'idée que le temps de la formation et de l'acquisition des savoirs de base n'est pas circonscrit à une période de la vie, suppose un processus continuel et répond ainsi parfaitement à l'exigence de flexibilité et d'adaptabilité des individus et de leur parcours. Les modalités de valorisation de l'expérience individuelle, qui vont jusqu'à la possibilité de certification et offrent une alternative au suivi de formations, participent aussi de cette incitation à s'adapter ainsi qu'à créer sa propre activité (entrepreneuriat).

L'un des effets pervers de cette logique de flexibilité, c'est qu'elle génère des risques et un sentiment d'insécurité. Le terme et concept de flexicurité (Vergnies, 2011) ou celui de sécurisation des parcours professionne $/ s^{4}$ reflètent une volonté politique paradoxale de protéger l'emploi sans remettre en question cet environnement instable, de ménager de nouvelles sécurités dans un contexte dont on promeut et légitime l'instabilité et l'imprévisibilité. Cette ambition paradoxale se répercute sur le statut de la formation. Censée aider les salariés à entretenir leurs compétences et leur «employabilité» de manière à leur permettre de retrouver un emploi le plus vite possible dès lors que celui qu'ils occupent aura disparu, elle ne peut en fait garantir un genre de sécurité équivalent à celle que procurait l'occupation de longue durée d'un emploi.

\section{Redéfinitions du rapport au temps et à la distance dans l'activité}

L'analyse scientifique du travail supposait selon Taylor la possible mesure du temps de réalisation de l'activité.

4 Travail et emploi, $\mathrm{n}^{\circ} 113$, janvier 2008.
Aujourd'hui encore, le contrôle et l'appréciation de I'activité sont toujours ancrés dans ce rapport au temps considéré comme optimum pour la réaliser. Les débats sociaux sur la réduction, l'allongement, la mesure du temps de travail ont été nourris et récurrents pendant ces dernières décennies. Ils sont restés prisonniers d'une conception associée au travail posté, dans un contexte spatio-temporel facile à circonscrire. Ils n'ont en tout cas presque jamais intégré la compréhension du travail actuel, le plus souvent très difficile à délimiter dans un temps et un contexte donnés, pas toujours là où il devrait être et qui ne se déroule pas toujours au moment où il le devrait. Les délocalisations de l'activité ou le déracinement des travailleurs appelés à changer d'environnement de travail, voire de domaine d'activité, impliquent globalement une distance de l'activité au territoire dans lequel l'acteur et les organisations ont leur histoire. Avec le développement des formations à distance, c'est aussi la formation qui s'inscrit dans un lieu devenu inconnu et quelquefois très éloigné.

En parallèle, le rythme des échanges sociaux et des activités semble s'accélérer, jusqu'à l'emballement, soumis à des exigences de juste-à-temps, de rapidité, de réactivité et de créativité dans la réactivité. Or, c'est la mise à distance («temporelle - suspension de l'urgence -, affective - suspension du jugement -, émotionnelle - suspension de la pression sociale "; Zeitler, Guérin \& Barbier, 2012) qui produit la réflexivité. Le processus d'accélération associé à cet empêchement à s'arrêter et à faire retour sur l'activité est un trait marquant de notre société alors même que les bienfaits associés à l'attitude réflexive y sont fortement valorisés.

\section{Individus et collectif dans l'activité redéfinie}

Les discours politiques et managériaux appellent de leurs vœux le développement d'une culture de l'autonomie, de la compétence, de la prise de risque et de la responsabilité. L'individu devrait « trouver en lui-même et en lui seul, en permanence, les ressources nécessaires pour accomplir sa tâche» (Linhart, 2009, p. 130). C'est ainsi que seraient évaluées sa compétence, son employabilité. L'individu ne s'inscrit plus dans une perspective collective à long terme, mais dans la gestion de son propre parcours professionnel (Guichard, 2009). Les orientations et les étapes de carrières sont de plus en plus incertaines. Le caractère spécifique des trajectoires se généralise. L'individualisation en marche 
est encore un vecteur d'accroissement des risques. «Une des caractéristiques de la société singulariste [serait] d'ailleurs la capacité relativement inédite des acteurs à trouver des solutions personnelles à des problèmes collectifs» (Martuccelli, 2010, p. 105). L'injonction à se prendre en charge interroge la fonction des collectifs. Comment être le sujet de son expérience sans les autres? Pourquoi l'évaluation des compétences, la valorisation de l'expérience et la capacité à créer son activité se font-elles quasi uniquement sur un registre individuel, sinon pour comparer et mettre en concurrence les acteurs? Si on peut parfois constater le recul du collectif dans le travail, il va pourtant de pair avec celui de la montée en puissance du rôle des réseaux, du travail en réseaux et des échanges communicationnels dans les réseaux virtuels par les moyens électroniques, autre paradoxe de notre rapport à l'activité. Alors que les solidarités au travail perdraient du terrain, la mise en visibilité des territoires intimes de I'activité humaine n'a jamais été aussi forte. Histoires de vie et parfois de travail se dévoilant sans pudeur dans les émissions de téléréalité. C'est donc plutôt le changement du rapport que l'individu entretient avec son activité qu'il faut remarquer plutôt que la disparition du collectif.

\section{Quête d'adéquation entre formation et emploi, professionnalisation, compétences et évaluation}

Formation et activité de travail ne peuvent être considérées et par conséquent analysées séparément, car la finalisation de la formation tient à l'heure actuelle dans la plupart des cas à l'usage et l'utilité qu'elle est destinée à avoir pour et dans l'activité (Barbier, 2001). La formation a donc pour fonction d'adapter les individus à leurs activités sociales, de leur permettre de trouver leur place et de jouer un rôle dans la société, de contribuer, par l'acquisition de savoirs et de compétences, à la compétition économique mondiale et à la création de valeur. Trois intentions sociales portent les reconfigurations de la relation que nous étudions : la quête de l'adéquation formation/emploi détermine l'intention sociale de professionnalisation qui elle-même met en œuvre la «logique» des compétences et le besoin social de connaissance et contrôle du travail.

\section{Une « incontournable professionnalisation »}

L'injonction sociale de professionnalisation signale le projet de mettre en adéquation formation et emploi. La montée en puissance de l'exigence de professionnalisation va de pair avec l'importance croissante accordée à l'ajustement entre les individus, leurs compétences et les besoins d'emploi (c'est-à-dire la place qui leur est assignée) dans la société. Du coup, les politiques, les dispositifs et même jusqu'aux outils destinés à aller dans le sens d'une professionnalisation des activités, des acteurs et des organisations s'inscrivent plus ou moins dans cette logique et cet habillage adéquationniste (Maillard, 2013) qui manifeste les paradoxes et les hypocrisies sociales sous-jacentes dans les intentions sociales de professionnalisation. Le chômage des jeunes et la nécessité pour les gouvernements successifs d'aménager, sans pouvoir l'annoncer de façon explicite, des modalités de «rétention » des jeunes en amont de l'emploi et d'allongement des transitions entre formation et emploi peuvent expliquer aussi bien l'évolution des diplômes professionnels (CAP, BEP, bac pro) que la démocratisation de l'accès à l'université ou encore l'incitation à l'entrepreneuriat des jeunes. Le problème non dépassé du chômage de certaines catégories de la population agit de manière puissante sur les discours, les politiques et les pratiques. En l'absence de véritable mouvement de création d'emplois, le développement de l'employabilité par la formation a peu d'effets sur le chômage... Mais, comme on le verra dans la troisième partie, des alternatives à la formation traditionnelle sont envisagées pour sortir de l'impasse.

\section{La logique des compétences}

Un des termes omniprésents aux articulations des champs du travail et de la formation est celui de compétence. Le fait de parler de logique ou d'approche compétence indique clairement pour le moins un modèle d'organisation, sinon un modèle de pensée qui tend, selon beaucoup d'analystes (Jonaert, 2009) à concurrencer celui de la qualification. L'usage du terme marque une transformation des rapports entre individus et organisation : «Avec la qualification, c'est le fait d'être qualifié qui vaut d'être reconnu; pour la compétence, c'est ici le fait d'être reconnu» (Lichtenberger, 1999, p. 84). Durrive montre comment des théories de l'action sont implicitement à l'œuvre dans les utilisations courantes du concept et comment le modèle de la compétence 
s'inscrit dans l'insécurité : «Entre qualification et compétence, l'organisateur aurait [...] à choisir entre inconfort (disposer d'une grille qui a tendance à le gêner dans sa répartition des rôles) et insécurité (se priver d'un garde-fou dans le rapport salarial pour mieux se rapprocher du travail réel ?)» (Durrive, 2013, p. 13).

\section{Le besoin social de connaissance et de contrôle du travail}

Le besoin de mieux connaître les activités de travail relève d'une quête de réassurance dans ce contexte d'insécurité et d'instabilité, donc de perte de repère. Les dimensions mal visibles du travail suscitent beaucoup d'intérêt chez les spécialistes de l'analyse du travail (Champy-Remoussenard, 2014), écho du besoin social de saisir les évolutions rapides et complexes du travail. Dans ce contexte, le recours à l'analyse de l'activité en tant que démarche scientifique constitue une caution de qualité, d'écoute des besoins sociaux et de prise en charge scientifique des problèmes. Les multiples motifs de recours à la mise en lumière du travail réel (Wittorski, 2014) expliquent la faveur qu'ont l'analyse du travail et ses diverses déclinaisons méthodologiques dans le champ de la formation (ChampyRemoussenard, 2005). Cette quête de repère génère de multiples entreprises pour formaliser ce qui ne l'était pas auparavant. Être un bon professionnel, c'est savoir le faire savoir, le dire, le manifester, exprimer l'expérience singulière qui caractérise et distingue tout un chacun. Dans une logique concurrentielle, le faire valoir, le faire savoir deviennent quasiment aussi importants que la détention du savoir et de l'expertise. En lien avec cet appel à la connaissance de l'activité et à sa formalisation, l'évaluation des activités est omniprésente (Dejours, 2003). Les transformations de l'activité en complexifient la compréhension et surenchérissent le besoin de contrôle et d'évaluation qui envahit tous les secteurs de l'activité, même ceux, là encore, qui étaient peu marqués par de telles logiques.

\section{Trois dispositifs analyseurs des reconfigurations des relations entre travail, éducation et formation}

L'analyse du développement des dispositifs éducatifs rapport éducation/formation/travail évolue, selon quels tensions et paradoxes, injonctions paradoxales et scénarios possibles en matière de statut donné à l'activité professionnelle. Les trois dispositifs choisis ici sont en voie de développement. Ils relaient les intentions politiques aux plans locaux, nationaux et internationaux en matière de transformation des champs du travail et des champs éducatifs. Ils se présentent comme des voies diversifiées vers la professionnalisation des activités éducatives et vers la recherche de l'adéquation formation/emploi. Ils s'inscrivent dans l'esprit du lifelong learning et d'un combat contre le chômage. Ils sont aussi autant d'exemples d'un appel à l'autonomie des acteurs et de formes d'individualisation des rapports sociaux. Après avoir quelquefois suscité le débat ou la surprise, ils font le plus souvent l'objet d'un consensus social. Cependant, ils ne se développent pas à la même vitesse et selon les mêmes logiques.

\section{La validation des acquis de l'expérience, une timide transformation des modalités d'accès à la certification}

En France, la législation sur la validation des acquis de l'expérience (VAE) $)^{5}$ inscrite au code du travail et de l'éducation, trouve en partie son origine dans des orientations politiques destinées à faciliter les mouvements de main-d'œuvre entre les États européens, les reconversions professionnelles et la mobilité des individus. L'accès aux titres et diplômes, par la voie de la VAE, va dans le sens de l'adaptation et de la fluidité de circulation des individus sur un marché du travail instable. II suppose également, en valorisant les acquis de l'expérience singulière, une individualisation très forte du rapport à l'accès au diplôme et à la qualification, conforme au déploiement de la logique des compétences.

La loi postule que l'expérience est formatrice et incite la société à la reconnaître comme telle. Elle fait l'objet d'une transaction sociale entre les individus et les organisations, permet d'accéder à la certification et est l'enjeu d'un ajustement dans la gestion de l'attribution des emplois et des salaires. En donnant cette valeur à l'expérience, la voie de la VAE donne un statut fort à l'activité et à l'activité professionnelle. Dans la mouvance des politiques éducatives européenne, la

5 Loi de modernisation sociale, 17 janvier 2002. Titre II, Travail, emploi et formation professionnelle, chap.II, développement de la formation professionnelle, section 3, VAE, art. 133-146. 
validation des acquis professionnels qui en était l'anticipation devient VAE en valorisant toute forme d'expérience et d'activité. Mais dans les pratiques, c'est bien le retour sur l'activité professionnelle qui fait l'objet de la plupart des demandes de certification, validant en cela le caractère central du travail.

En reconnaissant les acquis de l'expérience, qu'elle soit de travail ou d'une autre nature, au même titre que ceux de la formation, la législation sur la VAE déplace donc la hiérarchie qui caractérisait auparavant les rapports entre formation et travail. En transformant l'accès au diplôme, on peut supposer qu'elle puisse transformer les représentations que l'on s'en fait et modifier le rapport que les institutions et les professionnels chargés d'enseigner et de former entretiennent avec le travail.

Le retour sur l'activité sert bel et bien de voie d'accès au diplôme mais les études réalisées montrent qu'elle reste très marquée par la forme scolaire. En choisissant de recourir majoritairement à un dossier écrit, les organismes délivrant des certifications ont donné, en France, une place importante à la maîtrise de la langue écrite, laissant de côté des populations qui la maîtrisent mal (Presse, 2010). La professionnalisation limitée des nouvelles activités induites par la mise en œuvre de la VAE (accueil et accompagnement du candidat, participation à des jurys; Mayen \& Pin, 2013) n'a que faiblement transformé les pratiques des acteurs du système éducatif et celles des autres professionnels que l'évaluation du candidat est amenée à faire se rencontrer de manière inédite. Ceux qui sont impliqués sont d'ailleurs encore numériquement peu nombreux. L'impact de cette nouvelle modalité d'accès à la certification semble donc pour le moment limité en termes d'évolution du fonctionnement du système éducatif ou des modalités de recrutement et de gestion des ressources humaines. Tout en transformant de fait la relation entre expérience, activité et certification, les pratiques de VAE n'atteignent cependant pas certains segments des activités professionnelles, restent une voie secondaire d'accès à la certification et ne révolutionnent en rien les logiques dominantes de professionnalisation et de rapport aux compétences. Elles posent par ailleurs pour la énième fois le problème des inégalités d'accès à la formation et à la certification. Si elle légitime le statut de l'expérience et la pluralité des savoirs, la VAE reste un accès au diplôme, pivot solidement ancré dans la société française des rapports entre formation, emploi et rémunération.

\section{L'alternance et le développement des stages : un rêve adéquationniste?}

Des formes de formation dites «en alternance » se sont développées au cours des dernières décennies, proposant un modèle de formation partagé entre deux contextes, deux types d'acteurs et leur complémentarité d'action supposée. Ce développement apparaît clairement comme un des principaux modes de professionnalisation des formations et s'inscrit dans la logique compétences et la reconnaissance du potentiel formateur du travail. Plus encore que la formation en alternance à proprement parler et l'apprentissage, les stages en cours de période de formation se sont très fortement développés (Briant \& Glaymann, 2013, p. 20), mais «en dehors de tout cadre précis et pensé». La nécessité d'inclure un stage dans la formation apparaît comme un quasi-consensus social et son utilité est comme naturelle dans les représentations sociales. La question "à quoi sert le stage?» n'est que rarement véritablement posée, tant s'impose l'idée qu'il est aidant pour l'insertion professionnelle des diplômés et facteur d'amélioration de la pertinence et de la qualité de la formation. Récemment, le stage est devenu obligatoire dans les formations de l'enseignement supérieur. À la rentrée 2015 , dans le cadre de la loi sur la refondation de l'école, avec le PIODMEP (Parcours individuel d'information, d'orientation et de découverte du monde économique et professionnel, ou Parcours Avenir), l'Éducation nationale française intègre dans le secondaire les stages en entreprises dans la perspective de découverte des univers professionnels.

L'enseignement étant considéré comme insuffisamment réactif et insuffisamment adapté aux rapides évolutions des besoins en main-d'œuvre et aux enjeux de la compétition mondiale, la période de formation en entreprise apparaît alors, dans ses différentes déclinaisons (apprentissage, alternance, stages), comme une solution salvatrice et un signe d'entente mutuelle entre les milieux éducatifs et ceux des autres activités professionnelles. Elle veut participer de l'adaptation nécessaire de la population aux fluctuations de l'offre d'emplois disponibles. Mais le déficit en création d'emploi contrarie les espoirs associés aux efforts de professionnalisation des formations et contrarie la logique adéquationiste. Les recruteurs ont, compte tenu de la rareté des emplois disponibles, élevé leurs exigences en insistant sur le manque d'expérience des débutants. La concurrence est toujours plus forte entre les jeunes puisque le diplôme ne fait plus la différence. La catégorie des jeunes 
instruits inemployables croît en dépit du développement des stages et d'autres tentatives d'ajustement.

L'alternance et le stage impliquent un déplacement, une mobilité, une flexibilité, une oscillation entre les différents types de savoirs et les milieux dans lesquels ils circulent et se construisent et s'inscrivent bien dans les grandes tendances évoquées précédemment. Le va-et-vient entre ces deux contextes est plus ou moins précisément orchestré et, s'il nécessite ce qu'on a pu appeler une pédagogie du voyage (Lerbet-Séréni \& Violet, 1999), elle n'est pas toujours mise en œuvre. Le déplacement, qui est aussi détour, semble souvent suffire, gage de la professionnalisation. II relève aussi d'une épreuve, d'une expérience inédite pour le stagiaire mais aussi pour ceux qui en ont la responsabilité formative de part et d'autre. II implique de prendre certains risques, parfois même de partir à la dérive. La période en entreprise est supposée également répondre au déficit de réalité de la formation (cette réalité de l'activité de travail qui lui échappe). II s'agit alors d'aller voir, vérifier, légitimer les savoirs transmis, finalement de légitimer leur bien fondé et leur utilité sociale. Le stage veut rendre les savoirs opérants (Starck \& Deville, 2013). Mais, le principe de la formation en alternance ne garantit en rien la disparition du sentiment de fort décalage entre les enseignements reçus et les situations de travail rencontrées.

Ce décalage constitutif de la formation professionnelle, cette tension paradoxale, si souvent traduits de façon trop simpliste par l'opposition entre théorie et pratique pourrait être dépassés avec des dispositifs favorisant une production de connaissances sur l'activité et l'intégration, dans les formations en alternance, des stratégies de retour sur et d'analyse de l'expérience (Troger, 2000). Mais là encore, l'écart entre la quantité des stages et la qualité du retour sur expérience est grand. En se développant, les stages interpellent le rôle des tuteurs qui constitue une de leurs limites. Si dans certaines organisations du travail, la transmission fait de facto partie de l'activité (comme chez les artisans compagnons du devoir), ce n'est apparemment pas le cas partout. L'activité de tuteur et l'activité de travail sont de fait souvent concurrentes, au moins en matière de rapport au temps requis. L'exigence de formalisation de l'activité de tutorat dont s'assortissent souvent désormais les dispositifs ne fait qu'aiguiser ces difficultés. L'alternance n'a donc, pas plus que la VAE, une place naturelle dans la vie sociale. Elle s'y trouve plutôt injectée sur la base d'une forme d'illusion de réparation sociale des limites du système actuel.
La portée de la formation incluant des stages dans le moyen et le long terme reste une question peu débattue. Les résultats doivent être rapides, les profils modelés sur une réalité ponctuelle (Briant \& Glaymann, 2013, p. 27). Le stage s'ajuste aux besoins du moment, et pourtant les injonctions à la formation sont bien plus paradoxales : «On demande d'un côté aux jeunes entrant dans la vie active (et à ceux qui les forment) d'être immédiatement adaptés aux postes pour lesquels on veut les recruter et de l'autre d'être capables d'évoluer dans une vie professionnelle faisant appel à la flexibilité et à la mobilité » (Briant \& Glayman, 2013, p. 27). Pire, préparation supposée à l'emploi, le stage peut figurer comme un concurrent de l'emploi. Cet effet est gênant pour le consensus social qu'on a pointé plus haut. Et il peut arriver et arrive déjà que, tout comme l'emploi, le stage fasse défaut.

Les modèles du stage et de l'alternance (Durand, 2012) comme moyens actuels de professionnalisation et de formation reposent sur le principe du changement du contexte d'exercice habituel de l'activité qui souligne le rôle de l'environnement dans la construction des expériences. Le contexte quand il est inhabituel fait recomposer les habitudes et le vécu de situations, et le processus de déplacement est promu comme modèle de construction de l'expérience sans que les effets en soient vérifiés pas plus que les tensions paradoxales dans lesquelles ils mettent parfois les acteurs (Starck \& Deville, 2013). Cette forme d'articulation entre milieux des pratiques professionnelles et milieux éducatifs manifeste l'importance accordée au potentiel formateur de l'activité, l'interpellation diffuse et larvée de la forme scolaire, le mouvement vers plus de capacité de mobilité et d'adaptation, I'adhésion à la logique des compétences de l'ici et maintenant. Ce développement est à la fois puissant et inabouti dans ses modalités de mises en œuvre et eu égard à la mesure de ses effets. II relève d'un consensus social et d'un impensé tout à la fois.

\section{Vers un modèle entrepreneurial de l'activité et de la formation?}

Si le modèle entrepreneurial de l'activité est diffusé de plus en plus largement, il remonterait selon Vérin (2011, p. 3), qui a analysé la figure de l'entrepreneur et son évolution dans l'histoire, à l'entreprise chevaleresque, «première conception de l'action conquérante (dans laquelle le sujet se rend visible par des actes qui sont autant d'expressions de sa seule puissance d'agir)». Actuelle- 
ment, il est proposé comme modèle ou alternative consensuelle à d'autres formes d'emploi et défini par I'Europe comme objectif éducatif. En fait, l'engouement actuel pour le développement de l'entrepreneuriat s'inscrit dans des tentatives pour résoudre les problèmes d'emploi et de compétitivité rencontrés par de nombreux États. Nous faisons ici l'hypothèse qu'il participe logiquement tout à la fois de velléités et décisions politiques pour introduire de nouveaux modèles de fonctionnement sociaux et un nouveau rapport à l'activité professionnelle, voire à l'action, comme «épreuve immédiate de soi dans l'action risquée comme modalité d'insertion dans l'ordre social» (Vérin, 2011, p. 63).

Les motifs de développement de l'éducation à l'entrepreneuriat sont relatifs à des enjeux sociaux, comportementaux, éducatifs et d'orientation qui semblent faire consensus dans la classe politique au point qu'ils sont traduits, aux niveaux transnationaux ${ }^{6}$, nationaux et locaux, en compétences-clés au niveau européen (esprit d'initiative et d'entreprise), dans le socle commun des compétences français (autonomie et initiative), et enfin diversement déclinés dans des orientations régionales ${ }^{7}$. Les politiques préconisent le développement d'une culture de l'entrepreneuriat (Leger-Jarniou, 2012) qui englobe l'esprit d'initiative, l'esprit d'entreprendre et d'entreprise, présentés comme indispensables autant aux entrepreneurs (créateurs et chefs d'entreprises indépendants) qu'aux intrapreneurs (salariés mais mis au défi de mobiliser eux aussi ce type de rapport à l'activité) et globalement à tous les citoyens dans leurs diverses activités. L'esprit d'initiative (et d'autonomie) était envisagé dans le socle commun des compétences en France, en 2013, comme la capacité de «passer des idées aux actes, [...] l'envie de prendre des initiatives, d'anticiper, d'être indépendant et inventif dans la vie privée, dans la vie publique et plus tard au travail», de "concevoir, de mettre en œuvre et de réaliser des projets individuels ou collectifs dans les domaines artistiques, sportifs, patrimoniaux ou socio-économiques ${ }^{8}$. L'esprit d'entreprendre est

6 La compétence à entreprendre a ainsi été identifiée comme compétence de base au sommet de Lisbonne en 2000 et reprise par l'agenda d'Oslo en 2006.

7 Comme dans la SRIE (Stratégie régionale initiative entrepreunariat) et le label La Suite dans les idées, si on prend l'exemple du NordPas-de-Calais.

8 «Socle commun des compétences». Loi n²013-595 du 8 juillet 2013 d'orientation et de programmation pour la refondation de l'école de la République (article 4). En ligne: <http://www.education. gouv.fr/cid2770/le-socle-commun-de-connaissances-et-decompetences.html> (consulté le 10 octobre 2015). défini de façon assez proche par les institutions européennes. L'esprit d'entreprise est tantôt synonyme d'esprit d'entreprendre tantôt d'état d'esprit favorisant l'exercice du chef d'entreprise ou même de l'ensemble des acteurs qui y sont impliqués ${ }^{9}$.

En France, après un développement sans véritable pilotage central, cet objectif a été récemment présenté comme une priorité, après le discours du président François Hollande et les Assises de l'entrepreneuriat de 2013. Il a été annoncé qu'à tous les niveaux d'enseignement, l'esprit d'entreprendre devait être enseigné. Au début de l'année 2014, ce programme n'était cependant pas encore mis en œuvre de manière systématique sauf dans l'enseignement supérieur. Le gouvernement a en effet lancé les appels à projets PEPITE (Pôle étudiant pour l'innovation, le transfert et l'entrepreneuriat) destinés à organiser un maillage territorial permettant l'accompagnement et la formation des étudiants et jeunes diplômés qui souhaitent créer des entreprises, puis la mise en œuvre du statut national de l'Étudiant entrepreneur et du Diplôme universitaire d'étudiant entrepreneur. Au niveau du secondaire, si le rapportdu CNEE (Conseil nationaléducation-économie) annonce qu'il faut développer l'esprit d'entreprendre, les dispositifs antérieurement mis en place tels que les mini-entreprises ${ }^{10}$ continuent à exister en marge des curricula comme c'est le cas depuis de nombreuses années.

Le développement du modèle entrepreneurial montre comment les décisions politiques tendent à redéfinir le rapport à l'activité et à l'emploi des populations en s'appuyant sur les politiques éducatives. En effet, le modèle d'activité préconisé s'inscrit parfaitement dans les tendances d'évolutions commentées précédemment. Il va de pair avec une société du risque, flexible, qui exige des individus qu'ils soient adaptables, autonomes, mobiles, acceptent les risques, l'échec et soient indéfiniment capables de «rebondir » par de nouvelles initiatives. II signale le passage du modèle fordiste du salariat vers le statut de travailleur indépendant et poursuit le mouvement de remise en cause des régulations collectives du marché du travail. L'activité se déploie alors au fil des initiatives, des échecs et des success stories... Le développement « des

9 En la matière, ni les termes ni les définitions ne sont stabilisés en dépit des tentatives des institutions européennes.

10 Commission européenne (2005), Les mini-entreprises dans l'enseignement secondaire. Rapport final du groupe d'experts, projet "Procédure Best». 
éducations à l'entrepreneuriat » (Starck, 2013) assurerait l'opérationnalisation d'une société de la connaissance - ou plutôt son prolongement - dans une société de l'initiative et de la créativité entrepreneuriale, cette évolution assurant le passage d'une «économie managériale » à une «économie entrepreneuriale » (Capron, 2009).

Dans le même temps, la notion d'entreprise et sa conception même sont interrogées. Le rapport au travail et à l'emploi (Segrestin, Roger \& Vernac, 2014) est mis en débat. L'entrepreneuriat social et solidaire se développe comme alternative éthiquement acceptable, de nouveaux concepts d'entreprise émergent, comme celui d'entreprise à objet social étendu (Segrestin \& Hatchuel, 2012)11 ainsi que des formes juridiques d'entreprises adaptées aux enjeux de l'innovation.

Le fait que le mode entrepreneurial de l'activité soit proposé comme modèle marque une étape de plus dans le déploiement de la logique compétences. Mais, dans ce cas, il ne s'agit plus de préparer au métier, ni même aux compétences, mais à la capacité à imaginer de nouvelles activités (à la créativité) et à les concrétiser. La logique qualification y perd définitivement sa légitimité. Et l'entrepreneuriat implique une logique de professionnalisation qui s'écarte tout à la fois du modèle des professions et du modèle des métiers. En effet, si l'on considère que l'on forme des créateurs d'entreprise, le terme de métier ne peut plus être utilisé, dans la mesure où il est des créateurs d'entreprises si différents les uns des autres qu'on peine à les faire entrer dans la même catégorie. Les traits de l'entrepreneur révèlent les valeurs de l'organisation sociale. Le pluriel est incontournable. II n'y a pas un entrepreneur mais des entrepreneurs, des façons d'entreprendre, des entreprises, des esprits d'entreprendre et d'entreprise. Comment trouver dans ce cas des principes communs d'éducation et de formation?

D'une certaine manière, le modèle de l'entrepreneuriat remet en cause le modèle de la formation comme préparation à l'exercice d'une activité qui peut être préalablement décrite. C'est au contraire par une intervention éducative destinée à développer un potentiel qu'on met en place les conditions d'une création infinie d'activités nouvelles, terreaux d'occupations sociales et professionnelles, d'emplois et de "valeur»... Si la catégorie des compétences "fonctionne » toujours, dans ce modèle, l'accent est mis sur

11 Les auteurs proposent un statut juridique qui puisse intégrer d'autres buts que le seul profit. les compétences transversales et même les aptitudes. Éduquer à l'entrepreneuriat depuis le plus jeune âge, c'est transmettre une culture (Pepin, 2011) et des compétences qui, loin de constituer de seuls atouts pour s'inscrire dans le monde de l'entreprise, sont considérées comme des valeurs éducatives en soi et des acquis précieux pour tout individu, que ce soit dans l'exercice de sa profession ou dans sa vie quotidienne (OCDE, cité par Champy-Remoussenard, 2012).

Préconisé, prôné, soutenu, le mode entrepreneurial de l'activité prend forme et s'actualise dans les initiatives effectives des individus appelés à créer leur activité et responsables de leur destin professionnel et personnel. «Au moins, je travaille pour moi» est un propos courant d'entrepreneur. La création individuelle n'est cependant pas toujours individuelle. Les entrepreneurs s'appuient sur des réseaux, voire des solidarités familiales. Les compétences entrepreneuriales incluent l'esprit d'équipe ${ }^{12}$, et les pédagogies - comme les mini-entreprises - visant le développement de l'esprit d'entreprendre sollicitent les modes de travail en équipe (Champy-Remoussenard, Strark, Deville et al., 2014). Ce mode d'implication dans l'activité entre en résonance avec les tensions que nous avons caractérisées entre un travail hyper individualisé et un irréductible recours au collectif.

En conclusion, le modèle entrepreneurial est susceptible de faire évoluer considérablement les paramètres habituels de la relation éducation/formation/ travail. II entre en contradiction avec le modèle traditionnel de la transmission du travail et du métier et induit un bouleversement du rapport aux compétences. En effet, chaque entrepreneur crée une configuration d'activités inédite ou partiellement inédite, mobilisant des savoirs et des compétences eux aussi chaque fois différents et combinés différemment. On ne peut plus dans ce cas parler d'adéquation entre l'activité et un diplôme, envisager un référentiel d'activité et de compétences puisque l'activité est par essence singulière et doit l'être pour être légitime et viable. En cela le régime entrepreneurial de l'activité est à l'image de la société singulariste et du risque.

Nous ferons donc l'hypothèse qu'il est, dans une bien plus forte mesure que les deux autres dispositifs, le signal de transformations majeures du rapport à l'activité professionnelle et à la formation. L'«acculturation par l'action aux logiques de l'entreprise figurerait ainsi

12 Référentiel de compétences entrepreneuriales, Entrepreneuriat \& Esprit d'entreprendre. 
comme un nouveau repère pour se construire une représentation du monde et à terme arbitrer dans les choix d'orientation disponibles » (Starck, 2013, p. 8).

Le symposium de Montpellier ${ }^{13}$ a mis en évidence le potentiel d'interpellation de l'ordre scolaire établi que recèle l'éducation à l'entrepreneuriat (notamment Lebeaume, 2012; Audigier, 2012). Les formes prises dans l'école peuvent être étudiées dans cette perspective : les processus de résistance, de militance, les stratégies de contournement, de détournement, etc.; le rôle des enseignants volontaires, impliqués ou spectateurs; les modalités pédagogiques (pédagogies du projet, de l'action, de l'expérience), I'insertion dans les curricula en relation avec la forme scolaire. Finalement l'éducation à l'entrepreneuriat, comme les autres « éducations à », est peut-être un trublion du système éducatif et un des fers de lance d'une nouvelle forme scolaire. Celle qui prévaut est encore largement adaptée à la société industrielle et organisée en disciplines d'enseignement relativement figées alors qu'en parallèle des logiques éducatives relevant des nouvelles compétences-clés et des "éducations à» tentent de prendre en compte les besoins vitaux de notre société.

\section{En conclusion}

Finalement, on peut considérer que les dispositifs étudiés seraient les prototypes sociaux d'une acculturation sociale à de nouvelles formes de division des fonctions éducatives et à un nouveau rapport à l'activité. On s'acheminerait vers une nouvelle forme éducative de la société et une nouvelle forme scolaire adaptée à des activités de travail métamorphosées. Mais si, par certains aspects, les deux premiers dispositifs s'écartent de la forme scolaire, c'est cependant en y adhérant toujours partiellement. Pour l'heure, le système éducatif et de formation est encore peu modifié par la VAE et l'alternance, qui pourtant portent en germe de profonds bouleversements des rapports entre éducation, formation et travail. Seule peut-être la dynamique entrepreneuriale s'avère un vecteur puissant de transformations dont la portée n'est actuellement ni maîtrisable ni mesurable.

L'activité professionnelle et la formation sont au cœur d'un maelstrom de tensions, de paradoxes et d'injonctions paradoxales adressées aux individus, qui constituent autant d'analyseurs des changements en cours et signalent un certain désarroi social, associé aux défis des modèles de l'activité instable, précaire, de la mobilité et de l'adaptabilité. La complexité des évolutions en jeu et leur portée possible rendent nécessaire une analyse de nature politique des activités et des dispositifs et une attention particulière au choix des mots qui les désignent, agissant comme des révélateurs des paradoxes et tensions sociales à l'œuvre dans les dispositifs et vécus par les acteurs.

Le travail est tout à la fois un instrument d'émancipation ou d'asservissement. Les formes d'instabilité actuelles rendent possible le renouveau en même temps qu'elles font courir le risque du «déracinement » que $\mathrm{S}$. Weil a analysé dans ses ultimes écrits. D'après elle, l'homme reçoit «la presque totalité de sa vie morale, intellectuelle, spirituelle, par l'intermédiaire des milieux dont il fait naturellement partie : c'est le processus d'enracinement» (Weil, 1949, p. 61). S'enraciner c'est donner un sens à son milieu et éviter l'aliénation qui conduit à ne plus pouvoir ordonner le monde. Le rapport à l'activité permet-il actuellement encore l'enracinement dans la société, s'il est de fait marqué par l'angoisse (Sennett, 2006, p.49) suscitée par les bouleversements du rapport à l'activité? La déclaration de l'Organisation internationale du travail de Philadelphie de 1944 peut aider à réanalyser le sens de l'évolution des relations entre travail et formation : «le travail n'est pas une marchandise. Tous les êtres humains, quels que soient leur race, leur croyance ou leur sexe, ont le droit de poursuivre leur progrès matériel et leur développement spirituel dans la liberté et la dignité, dans la sécurité économique et avec des chances égales». Les risques d'une sorte de potentiel et de processus infini d'action, la promotion d'un modèle d'activité flexible et d'individus indéfiniment adaptables ne rend pas toujours possible l'enracinement. Pour que l'individu et les collectifs puissent se définir, se redéfinir, être créatifs, aboutir à un sentiment de complétude, l'action doit aussi s'enraciner...

Patricia Champy-Remoussenard Université de Lille, Lille 3, Proféor-CIREL patricia.remoussenard@univ-lille3.fr

13 Symposium intitulé «Premières discussions des recherches portant sur les objectifs et enjeux de l'éducation à l'entrepreneuriat» organisé dans le cadre du congrès AREF (Actualité de la recherche en éducation et formation), en août 2013. 


\section{Bibliographie}

ALBERO B. (2010). «La formation en tant que dispositif : du terme au concept ». In B. Charlier \& F. Henri, La technologie de l'éducation : recherches, pratiques et perspectives. Paris : PUF, p. 47-59.

AUDIGIER F. (2012). «Les éducations à... et la formation au monde social ». Recherches en didactiques, $n^{\circ} 14$, p. 47-64.

BACHELARD G. ([1934] 2000). La formation de l'esprit scientifique. Paris : Vrin.

BALIBAR É. (1968) «Sur les concepts fondamentaux du matérialisme historique». In L. Althusser \& É. Balibar (dir.), Lire «Le Capital». Paris : F. Maspero.

BARBIER J.-M. (2000). «Sémantique de l'action et sémantique d'intelligibilité des actions. Le cas de la formation ». In B. Maggi (dir.), Manières de penser, manières d'agir en éducation et en formation. Paris : PUF, p. 89-104.

BARBIER J.-M. (2001). «La formation des adultes, crise et recomposition ». In CRF-CNAM, Questions de recherches en éducation. Paris : INRP, p.11-28.

BARBIER J.-M. (2011). Vocabulaire d'analyse des activités. Paris : PUF.

BAREL Y. (1989). Le paradoxe et le système. Grenoble : Presses universitaires de Grenoble.

BECK U. (1986). La société du risque sur la voie d'une autre modernité. Paris : Flammarion.

BONGRAND P. (2012). «La mise en système et l'économicisation de l'enseignement en France au début des années 1950 : la fonctionnalisation d'une institution». Politix, vol.2, n98, p.35-56.

BRIANT (de) V. \& GLAYMANN D. (dir.) (2013). Le stage. Formation ou exploitation? Rennes: Presses universitaires de Rennes.

CAPRON H. (2009). Entrepreneuriat et création d'entreprises. Facteurs déterminants de l'esprit d'entreprise. Bruxelles : De Boeck.

CASTEL R. (2013). Les métamorphoses de la question sociale. Une chronique du salariat. Paris : Fayard.

CHAMPY-REMOUSSENARD P. (2005). «Les théories de l'activité entre travail et formation ». Savoirs, $n^{\circ} 8$, p. 9-50.

CHAMPY-REMOUSSENARD P. (2012). «L'éducation à l'entreprenariat : enjeux, statut, perspectives». Spirales, n50, p.39-51.

CHAMPY-REMOUSSENARD P. (dir.) (2014). En quête du travail caché : enjeux scientifiques, sociaux, pédagogiques. Toulouse : Octarès.

CHAMPY-REMOUSSENARD P., STARK S., DEVILLE J., AIT'MBARK M. \& ZAID A. (2014). Rapport de recherche Innovons et développons l'esprit d'entreprendre. Université Lille 3, CIREL.

CONTER B. \& ORIANNE J.-F. (2011). «La flexicurité et la formation des demandeurs d'emploi : les politiques wallonnes à l'aune de l'approche par les capacités». Formation emploi, $\mathrm{n}^{\circ} 113, \mathrm{p} .49-62$.

DEJOURS C. (2003). L'évaluation du travail à l'épreuve du réel. Critique des fondements de l'évaluation. Paris : INRA.
DELORY-MOMBERGER C. \& NIEWADOMSKI C. (2009). Vivre/ survivre. Récits de résistance. Paris : Téraèdre.

DURAND M. (2012) «L'alternance une métaphore prometteuse d'innovation sociale et éducative». Éducation Permanente, 2012/4, n 193, p.31-40.

DURRIVE L. (2013) Compétences et activité de travail. "L'art de gérer ses débats de normes». Synthèse de recherche en vue de I'HDR, université de Strasbourg, LISEC.

IMBERT P. \& DURAND M. (2014). «Utilitarisme et anti-utilitarisme dans le travail : pour une éthique en actes de l'éducation ». In P. Champy-Remoussenard (dir.), En quête du travail caché : enjeux scientifiques, sociaux, pédagogiques. Toulouse : Octarès, p.24-38.

GRILLOT T. (2013). «Le travail : valeurs, attentes et frustrations. Un entretien avec Robert Castel, Dominique Méda et Laurence Roulleau-Berger». La vie des idées. En ligne: $<$ http://www.laviedesidees.fr/Le-Travail-valeursattentes-et.html> (consulté le 10 octobre 2015).

GUICHARD, J. (2009). «Problematiche e sfide dell'orientamento nelle società industriali globalizzate all'inizio del XXI secolo». In A. Di Fabio (dir.), Career counseling e bilancio di competenze. Esperienze italiane e prospettive internazionali. Florence : Giunti Organizzazioni Speciali.

JONAERT P. (2009). Compétence et socioconstructivisme, un cadre théorique. Bruxelles : De Boeck.

LEBEAUME J. (2009). «Effervescence contemporaine des propositions d'éducations à... regard rétrospectif pour le courant curriculaire à venir». Spirales, n50, p.11-24.

LEGER-JARNIOU, C. (2012) «Développer la culture entrepreneuriale chez les jeunes». In M. Gomez-Breysse \& A. Jaouen (dir.), L'entrepreneur au $27^{e}$ siècle. Reflet des évolutions sociétales. Paris : Dunod, p. 123-140.

LERBET-SÉRÉNI F. \& VIOLET D. (1999). «Paradoxes de la médiation. Tradition et alternance». Revue française de pédagogie, $\mathrm{n}^{\circ} 128, \mathrm{p} .17-24$.

LICHTENBERGER Y. (1999). «Compétence, organisation du travail et confrontation sociale». Formation emploi, $n^{\circ} 67$, p. 93-107.

LINHART D. (2009). Travailler sans les autres? Paris : Éd. du Seuil.

MARIE P. (2011). La croyance, le désir, l'action. Paris : PUF.

MAILLARD F. (2013). «Les diplômes professionnels de l'éducation nationale entre scolarisation et professionnalisation ». Les Dossiers des sciences de l'éducation, n³0, p. 35-52.

MAYEN P. \& PIN J.P. (2013). «Conditions et processus de l'engagement en VAE ». Formation emploi, n²16, p.13-29.

MARTUCELLI D. (2010). La société singulariste. Paris : Armand Colin.

MÉDA D. (1998). Le travail. Une valeur en voie de disparition. Paris : Flammarion.

MONCEAU G. (dir.) (2013). Le courrier électronique dans les pratiques professionnelles, en éducation, santé et action sociale : usages et effets. Paris : Champ social éd. 
NAVILLE P. (1954). La vie de travail et ses problèmes. Paris : Armand Colin.

NAVILLE P. (1963). Vers l'automatisme social? Paris : Gallimard.

PEPIN M. (2011). «L'entrepreunariat en milieu scolaire, de quoi s'agit-il?». McGill Journal of Education/Revues des Sciences de l'éducation de McGill, nº 46-2, p. 303-326.

PRESSE M.-C. (2010). «Le processus de reconnaissance en VAE des personnes faiblement scolarisées : le rapport à la langue». TransFormations-Recherches en éducation des adultes, $\mathrm{n}^{\circ} 4$, p. 63-73.

SCHWARTZ Y. (2014) «Voir le travail». In P. Champy-Remoussenard (dir.), En quête du travail caché: enjeux scientifiques, sociaux, pédagogiques. Toulouse : Octarès, p.1-9.

SEGRESTIN B. \& HATCHUEL A. (2012). Refonder l'entreprise. Paris : Éd. du Seuil.

SEGRESTIN B., ROGER B. \& VERNAC S. (2014). L'entreprise, point aveugle du savoir. Paris : Éd. Sciences humaines.

SENNETT R. (2006). La culture du nouveau capitalisme. Paris : Albin Michel.

STARCK S. (2013). Éducation à l'entrepreneuriat : ressource pour s'orienter dans une nouvelle économie? Symposium "Orientation », congrès AREF 2013.

STARCK S. \& DEVILLE J. (2013). «Stage long en entreprise : un dispositif original qui interroge la professionnalité enseignante dans le monde du travail ». Les Dossiers des sciences de l'éducation, n³0, p. 85-99.

TROGER V. (dir.) (2000). «Les formations professionnelles entre l'École et l'Entreprise». Revue française de pédagogie, $\mathrm{n}^{\circ} 131$.

VERGNIES J.-F. (2011). «Édito : Flexicurité sans capacité n'est que ruine de l'homme», Formation emploi, n¹13, p.1-2.

VÉRIN H. (2011). Entrepreneurs, entreprise. Histoire d'une idée. Paris : Classiques Garnier.

WEIL S. (1949). L'enracinement. Prélude à une déclaration des devoirs envers l'être humain. Paris : Gallimard.

WITTORSKI R. (2008). «La professionnalisation». Savoirs, $\mathrm{n}^{\circ} 17$.

WITTORSKI R. (2014). «Rendre visible la part insue de l'activité : quelques motifs, enjeux et fonctions remplies». In P. Champy-Remoussenard (dir.), En quête du travail caché: enjeux scientifiques, sociaux, pédagogiques. Toulouse : Octarès, p. 101-113.

ZARIFIAN P. (1995). Le travail et l'événement. Paris : L'Harmattan.

ZEITLER A., GUÉRIN J. \& BARBIER J.-M. (dir.) (2012). «La construction de l'expérience». Recherche \& formation, n 70 , p. 9-14. 